\title{
Homodimeric cross-over structure of the human granulocyte colony-stimulating factor (GCSF) receptor signaling complex
}

\author{
Taro Tamada*, Eijiro Honjo ${ }^{\dagger \ddagger}$, Yoshitake Maeda ${ }^{\dagger}$, Tomoyuki Okamoto $^{\dagger}$, Matsujiro Ishibashi ${ }^{\S}$, Masao Tokunaga ${ }^{\S}$, \\ and Ryota Kuroki*ף
}

\begin{abstract}
A crystal structure of the signaling complex between human granulocyte colony-stimulating factor (GCSF) and a ligand binding region of GCSF receptor (GCSF-R), has been determined to $2.8 \AA$ resolution. The GCSF:GCSF-R complex formed a 2:2 stoichiometry by means of a cross-over interaction between the Ig-like domains of GCSF-R and GCSF. The conformation of the complex is quite different from that between human GCSF and the cytokine receptor homologous domain of mouse GCSF-R, but similar to that of the IL-6/gp130 signaling complex. The Ig-like domain cross-over structure necessary for GCSF-R activation is consistent with previously reported thermodynamic and mutational analyses.
\end{abstract}

*Research Group for Molecular Structural Biology, Quantum Beam Science Directorate, Japan Atomic Energy Agency, 2-4 Shirakata-Shirane, Tokai, Ibaraki 319-1195, Japan; †Pharmaceutical Research Laboratory, Kirin Brewery Co. Ltd., 3 Miyahara-cho, Takasaki 370-1295, Japan; and \$Laboratory of Applied Microbiology, Faculty of Agriculture, Kagoshima University, 1-21-24 Korimoto, Kagoshima 890-0065, Japan

\section{Results}

ligand-receptor interaction | x-ray crystallography | IL-6 | gp130

G ranulocyte colony-stimulating factor (GCSF) has become an important cytokine for medical treatment of patients suffering from granulopoenia (1-3) through regulating the maturation, proliferation, and differentiation of the precursor cells of neutrophilic granulocytes. The mature GCSF receptor (GCSF-R) is composed of an extracellular region, which consists of an Ig-like domain, a cytokine receptor homologous $(\mathrm{CRH})$ domain, three fibronectin type III-like domains, a transmembrane region, and a cytoplasmic domain (4). The CRH domain is further divided into an N-terminal $(\mathrm{BN})$ and a $\mathrm{C}$-terminal (BC) domain containing a WSXWS sequence motif that is highly conserved in the CRH domain of cytokine receptors (5).

Binding of GCSF to the extracellular Ig-like and CRH domain of its receptor triggers receptor homodimerization, resulting in activation of Janus tyrosine kinase-signal transducers and activators of transcription (JAK-STAT) type signaling cascades (6). The stoichiometry of the GCSF/GCSF-R complex has been a matter of some debate, with various proposed values $(1: 1,2: 2$, and/or 4:4) (7-9). Our recent report demonstrated that only the $2: 2$ stoichiometry is observed as a stable complex (10).

The crystal structure of a 2:2 complex between human GCSF (hGCSF) and the CRH domain of mouse GCSF-R has been reported (11). Although this structure provided important details of ligand-receptor interactions, it was not able to clearly elucidate the activation mechanism of GCSF-R because the complex did not include the natural Ig-like domain with high affinity for ligand (12).

Here, we report the crystal structure of a complex between hGCSF and the Ig-like and CRH (Ig-CRH) domains of human GCSF-R (hGCSF-R) at 2.8- $\AA$ resolution. The signaling 2:2 complex is formed by means of cross-over interactions between the Ig-like domain of hGCSF-R and the neighboring hGCSF, forming a twofold axis of crystallographic symmetry. This conformation is quite different from that of the heterogeneous mouse GCSF-R complex and more closely resembles the 2:2:2 active assembly of human interleukin-6 (hIL-6), hIL-6 $\alpha$-receptor (hIL-6R $\alpha$ ), and human gp130 [which is a shared signal transducing receptor for several cytokines (13)], and the 2:2 assembly of viral IL-6 (vIL-6) and human gp130 (14).
Overall Structure. The 2:2 complex between hGCSF and hGCSF-R is present as the association of two 1:1 complexes by means of a twofold crystallographic axis of symmetry (Fig. 1). The location of secondary-structure elements within the primary structure is shown in Fig. 2. The overall shape of the complex appears as an "unstable table" consisting of two diagonal "legs" (BC domain) and a "tabletop" with approximate dimensions of $97 \times 67 \times 60 \AA$. Each BC domain of hGCSF- $R$ in the 2:2 complex is situated parallel with a 68 - $\AA$ distance between the $\mathrm{C}$ termini of both $\mathrm{BC}$ domains. Two 1:1 complexes are tethered at each Ig-like domain of the receptor. The angle of $\mathrm{BN}$ and $\mathrm{BC}$ domains of Ig-CRH [defined between $\mathrm{C}^{\alpha}$ atoms of Gly-97 (first residue in BN) and Glu-308 (last residue in BC) through Leu-172] is $105^{\circ}$. The structure of the Ig-like domain comprising residues 3-96 contains eight $\beta$ strands and connecting segments (an Ig fold) and is connected to the BN domain with an inclination of $\approx 128^{\circ}$ (defined by three $\mathrm{C}^{\alpha}$ atoms of His-33, Gly-97, and Leu-172). Two disulfide bonds, Cys-3-Cys-29 and Cys-23-Cys78 , are observed in the Ig-like domain and conflict with a previous report of disulfide bridges involving Cys-3-Cys-78 and Cys-23-Cys29 , as determined by peptide mapping (15).

Ligand-Receptor Binding. From previous mutational analyses, it has been proposed that the receptor recognition of hGCSF is achieved through the major (site II) and the minor (site III) sites (16-18). The ligand-receptor interactions observed within $4.0 \AA$ are summarized in Table 1 and Fig. 3 (site II, red; site III, blue). The site II interaction consisting of the two GCSF helices $\alpha \mathrm{A}$ (Gln-11-Cys36) and $\alpha \mathrm{C}$ (Gly-100-Glu-122) includes Lys-16, Glu-19, Gln-20, Arg-22, and Lys-23 located in $\alpha \mathrm{A}$, and Leu-108, Asp-109, and Asp-112 in $\alpha \mathrm{C}$ interacting with the "bend" region of the $\mathrm{CRH}$ domain in the receptor [consisting of Arg-167, Leu-171, Leu-172, and Tyr-173 of BN domain, and His-238, Ile-239, Asn-240, Arg-288, and Leu-291 of BC domain (Fig. 3a)]. Ala mutations of Glu-19, Arg-22, Asp-109, and Asp-112 resulted in a decrease in biological activity (16-18). A total of seven hydrogen bond interactions were observed for the site II interaction (Table 1). The Ala mutations of Leu-172, Tyr-173, His-238, Asn-240, and Arg-288 in hGCSF-R also resulted in a decrease in biological activity (Table 1) (19). Besides

Conflict of interest statement: No conflicts declared.

Abbreviations: GCSF, granulocyte colony-stimulating factor; hGCSF, human GCSF; GCSF-R, GCSF receptor; hGCSF-R, human GCSF-R; CRH, cytokine receptor homologous; $\mathrm{mCRH}$ mouse $\mathrm{CRH}$; hIL-6, human IL-6; vIL-6, viral IL-6; hIL-6R $\alpha$, hIL-6 $\alpha$-receptor; BN, N-terminal domain of the $\mathrm{CRH}$ region; $\mathrm{BC}$, C-terminal domain of the $\mathrm{CRH}$ region.

Data deposition: The atomic coordinates have been deposited in the Protein Data Bank, www.pdb.org (PDB ID code 2D9Q).

₹Present address: Research Group for Molecular Structural Biology, Quantum Beam Science Directorate, Japan Atomic Energy Agency, 2-4 Shirakata-Shirane, Tokai, Ibaraki 319-1195, Japan.

ๆTo whom correspondence should be addressed. E-mail: kuroki.ryota@jaea.go.jp.

() 2006 by The National Academy of Sciences of the USA 


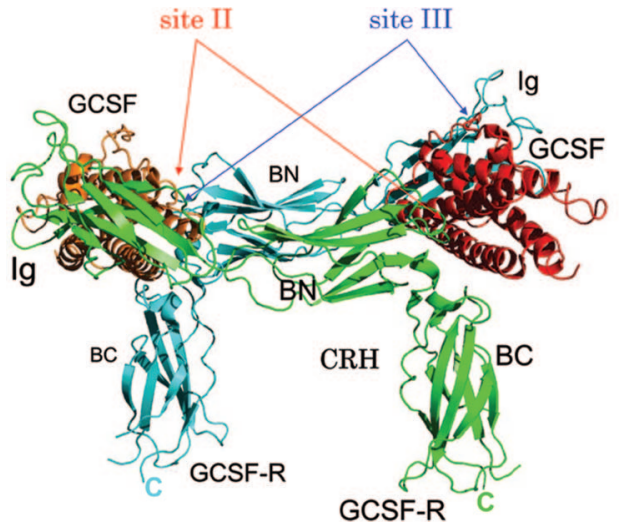

Lys-16, Gln-20, Lys-23, and Leu-108 in hGCSF and Leu-171, Tyr-173, Asn-240, and Leu-291 are involved in the site II interaction through van der Waals interactions (Table 1).

Site III of hGCSF, consisting of the N-terminal region in $\alpha \mathrm{D}$

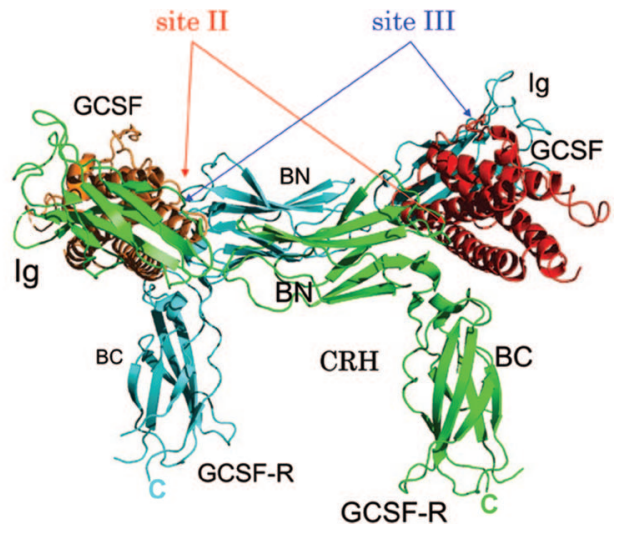

Fig. 1. Overall structure of the 2:2 complex between hGCSF and hGCSF-R (Ig-CRH domain). The GCSF molecules are colored in red and orange, and GCSF-R molecules are colored in green and cyan. Fig. 1 was prepared by the PYMOL Molecular Graphics System (DeLano Scientific, San Carlos, CA). a

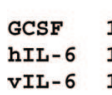

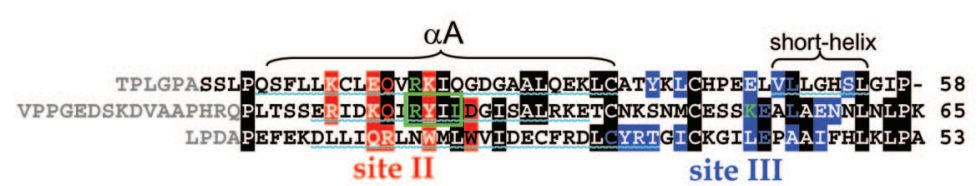

$\alpha B$
(Ala-143-Arg-169; including Phe-144 and Arg-147), and the A-B loop region (Ala-37-Gly-73; including Tyr-39, Leu-41, Glu-46, Val-48, Leu-49, and Ser-53) exhibit interactions with the Ig-like domain of another hGCSF-R chain (including Cys-3,

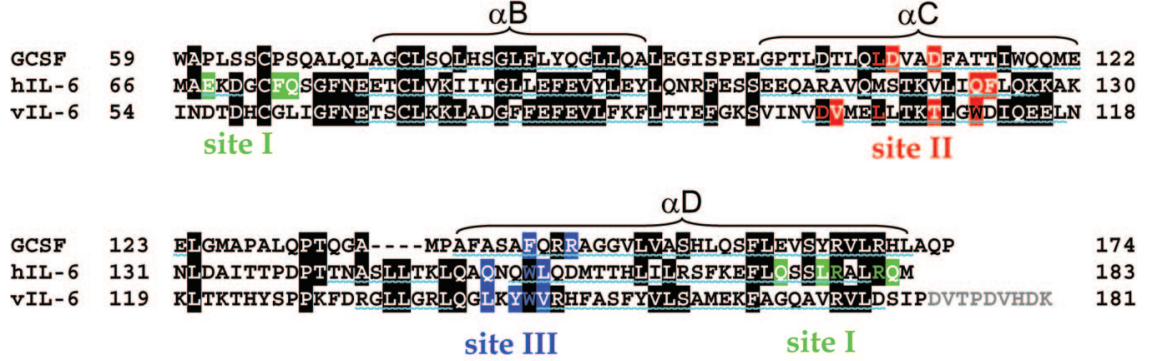

b

GCSF-R 2 gp130 1

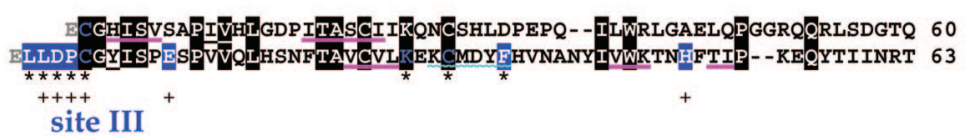

site III
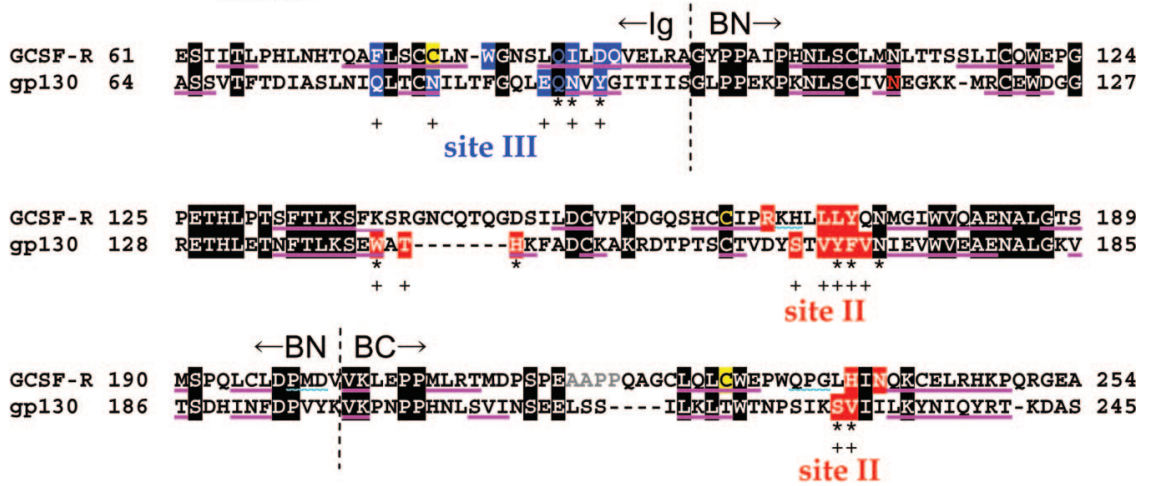

GCSF-R 255 SWWALVGPL--PLEALQYELCGLIPATAYTLOIRCIRWPLPGHWSDWSPSLELRTTERAA gP130 246 TWSOIPPEDTASTRSSFTVQDLKPFTEYVFRIRCMKEDGKGYWSDWSEEASGITYEDR- 303

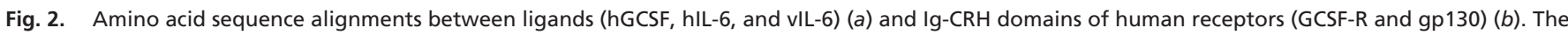

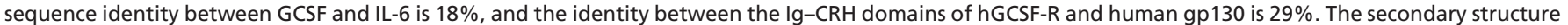

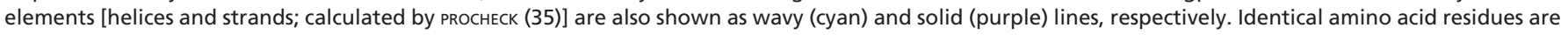

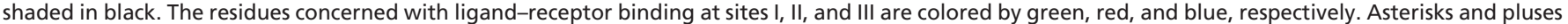

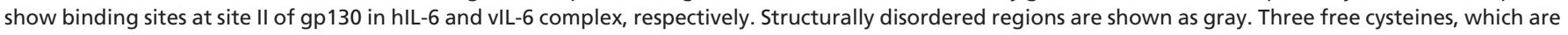
colored yellow, in Ig-CRH domains of GCSF-R are mutated to serines in this study. 
Table 1. Related residues of ligand-receptor binding

\begin{tabular}{|c|c|c|c|c|c|c|c|c|}
\hline \multirow[b]{2}{*}{ Site } & \multicolumn{2}{|c|}{ Ala mutations in ligand } & \multicolumn{3}{|c|}{ Interaction between ligand and receptor } & \multicolumn{3}{|c|}{ Ala mutations in receptor } \\
\hline & Residue & Proliferation* & Atom & Distance, $\AA$ & Atom & Proliferation* & Residue & Domain \\
\hline \multirow[t]{13}{*}{ II } & Lys-16 & $1.1^{\dagger}, 7.0^{\ddagger}$ & $\mathrm{C} \gamma$ & 3.99 & $\mathrm{C} \delta 2$ & - & Leu-291 & $\mathrm{CRH}$ \\
\hline & Glu-19 & $18^{\dagger}, 270^{\ddagger}, 9.6^{\S}$ & $\mathrm{O} \varepsilon 2$ & $2.25 \pi$ & $\mathrm{O}_{\eta}$ & $33^{\|}$ & Tyr-173 & \\
\hline & & $18^{\dagger}, 270^{\ddagger}, 9.6^{\S}$ & $\mathrm{O} \varepsilon 2$ & 3.47 & $C_{\gamma 1}$ & $59 \|$ & Ile-239 & \\
\hline & & $18^{\dagger}, 270^{\ddagger}, 9.6^{\S}$ & $\mathrm{O} \varepsilon 1$ & $2.58^{\natural}$ & $\mathrm{N} \eta 2$ & $280 \|$ & Arg-288 & \\
\hline & Gln-20 & $3.4^{\ddagger}$ & $\mathrm{C} \delta$ & 2.94 & $\mathrm{C} \delta 2$ & $33 \|$ & Tyr-173 & \\
\hline & Arg-22 & $0.9^{\dagger}, 3.7^{\ddagger}$ & $\mathrm{N} \varepsilon$ & $3.42^{\pi}$ & $\mathrm{O}$ & $11^{\|}$ & His-238 & \\
\hline & & $0.9^{+}, 3.7^{\ddagger}$ & $\mathrm{N} \varepsilon$ & $3.40^{\pi}$ & $\mathrm{O} \delta 1$ & $1.2^{\|}$ & Asn-240 & \\
\hline & Lys-23 & $1.1^{\dagger}, 20^{\ddagger}, 71^{\S}$ & $\mathrm{C} \beta$ & 3.13 & $\mathrm{~N} \delta 1$ & 1.2 & & \\
\hline & Leu-108 & $0.4^{+}$ & $\mathrm{C} \beta$ & 3.84 & $\mathrm{~N} \eta 1$ & - & Arg-167 & \\
\hline & Asp-109 & $15^{+}$ & $\mathrm{O} \delta 1$ & $2.91^{\pi}$ & $\mathrm{N} \eta 1$ & - & & \\
\hline & Asp-112 & $2.4^{\dagger}, 16^{\ddagger}, 2.1^{\S}$ & $\mathrm{O} \delta 1$ & $2.86^{\natural}$ & $\mathrm{N} \eta 2$ & - & & \\
\hline & & $2.4^{\dagger}, 16^{\ddagger}, 2.1^{\S}$ & $\mathrm{O} \delta 1$ & $3.12^{9}$ & $\mathrm{~N}$ & $5.6 \|$ & Leu-172 & \\
\hline & & $2.4^{+}, 16^{\ddagger}, 2.1^{\S}$ & $\mathrm{O} \delta 2$ & 3.79 & $\mathrm{C} \delta 1$ & - & Leu-171 & \\
\hline \multirow[t]{13}{*}{ III } & Tyr-39 & $1.8^{\dagger}$ & $\mathrm{C}_{\alpha}$ & 3.98 & $\mathrm{C} \zeta$ & $8.3 * *$ & Phe-75 & Ig-like \\
\hline & & $1.8^{+}$ & 0 & $3.16 \pi$ & $\mathrm{N} \varepsilon 2$ & $4.1 * *$ & Gln-91 & \\
\hline & Leu-41 & - & $\mathrm{C} \delta 2$ & 3.48 & $\mathrm{O} \varepsilon 1$ & $4.1 * *$ & & \\
\hline & Glu-46 & $2.5^{\dagger}, 5.7^{\ddagger}, 22^{\S}$ & $\mathrm{O} \varepsilon 2$ & 3.55 & $\mathrm{C} \delta 1$ & - & Ile-88 & \\
\hline & & $2.5^{\dagger}, 5.7^{\ddagger}, 22^{\S}$ & 0 & $3.03^{\pi}$ & $\mathrm{N}$ & - & & \\
\hline & & $2.5^{\dagger}, 5.7^{\ddagger}, 22^{\S}$ & $\mathrm{O}$ & $3.09 \pi$ & $\mathrm{N} \varepsilon 2$ & $14^{* *}$ & Gln-87 & \\
\hline & Val-48 & $3.0^{+}$ & $\mathrm{N}$ & $3.52^{9}$ & $\mathrm{O} \varepsilon 1$ & $14^{* *}$ & & \\
\hline & Leu-49 & $2.4^{+}$ & $\mathrm{C} \beta$ & 2.54 & $\mathrm{~N} \varepsilon 2$ & $14 * *$ & & \\
\hline & & $2.4^{+}$ & $\mathrm{C} \delta 2$ & 3.70 & $\mathrm{C} \delta 1$ & $2.6 * *$ & Trp-82 & \\
\hline & Ser-53 & $0.9^{+}$ & $\mathrm{C} \beta$ & 3.65 & $\mathrm{~S} \gamma$ & - & Cys-3 & \\
\hline & Phe-144 & $6.0^{+}$ & $\mathrm{C} \beta$ & 3.44 & $\mathrm{O} \delta 1$ & - & Asp-90 & \\
\hline & & $6.0^{+}$ & $\mathrm{C} \delta 1$ & 3.21 & $\mathrm{C} \beta$ & 4.1 ** & Gln-91 & \\
\hline & Arg-147 & - & $\mathrm{N} \eta 1$ & $2.86 \pi$ & $\mathrm{O} \varepsilon 1$ & $4.1 * *$ & & \\
\hline
\end{tabular}

*Value of proliferation shows ratio of $\mathrm{EC}_{50}$ of Ala-mutant/wild-type. - , no data.

${ }^{\dagger}$ Ref. 16.

${ }^{\ddagger}$ Ref. 17.

§Ref. 18.

"Hydrogen bond.

"Ref. 19.

** Ref. 20.

a

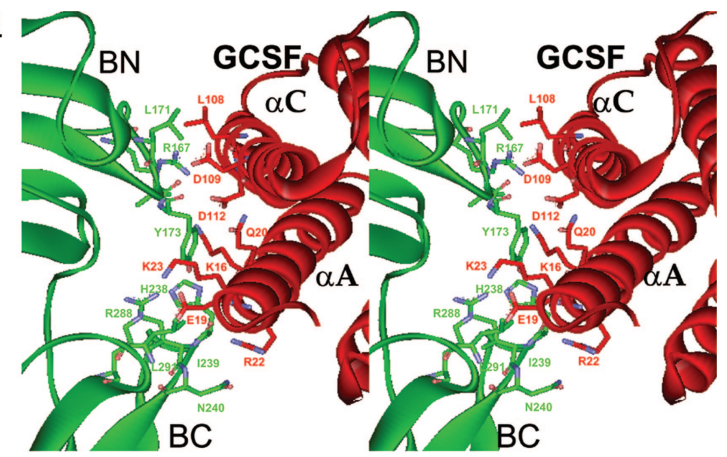

b

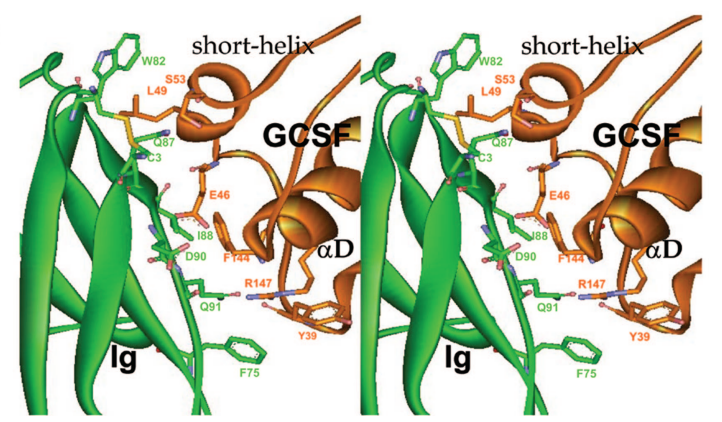

Fig. 3. Close-up view of the interface between ligand and receptor. (a) Site II. (b) Site III. Related residues of ligand-receptor binding are drawn as stick model. Figs. 3-5 were prepared by WEBLAB VIEWER LITE (Accelrys Inc., San Diego).
Phe-75, Trp-82, Gln-87, Ile-88, Asp-90, and Gln-91; Fig. 3b). The site III interaction primarily involves hydrophobic residues, Leu-41, Val-48, Leu-49, and Phe-144 in hGCSF and Phe-75, Trp-82, and Ile-88 in the receptor. The aromatic ring of Tyr-39 in hGCSF exhibits a stacking interaction parallel to that of Phe-75 in hGCSF-R. Five hydrogen-bonding interactions also contribute to the site III interaction as shown in Table 1. In these residues, Glu-46 and Val-48 in hGCSF and Phe-75, Gln-87, and Gln-91 in hGCSF-R are reported to be important for ligand binding and biological function $(16-18,20)$.

\section{Discussion}

The crystal structure of a complex between hGCSF and the Ig-CRH domains of hGCSF-R revealed a 2:2 complex formed by means of cross-over interactions between the Ig-like domain of hGCSF-R and the neighboring hGCSF (Figs. 1 and $4 a$ ). The 2:2 complex formed a twofold axis of crystallographic symmetry, although it is a quite different twofold axis from the crystal structure of the complex of hGCSF and mouse CRH (mCRH) domain (hGCSF/mCRH) previously determined (11) (Fig. 4b). The $\mathrm{mCRH}$ and human $\mathrm{CRH}$ (hCRH) domains exhibit similar structures (rms deviation $=1.4 \AA$ for $\mathrm{C}^{\alpha}$ atoms) for the region of the amino acid sequence exhibiting the greatest identity ( $64 \%$ identity from Ala-96 to Glu-308 in hGCSF-R). The structures of the GCSF-CRH complexes created through site II interaction are also quite similar between hGCSF/mCRH and hGCSF/ hCRH (rms deviation $=1.8 \AA$ for $\mathrm{C}^{\alpha}$ atoms). Although the $\mathrm{hGCSF} / \mathrm{mCRH}$ structure appears to be composed from two 

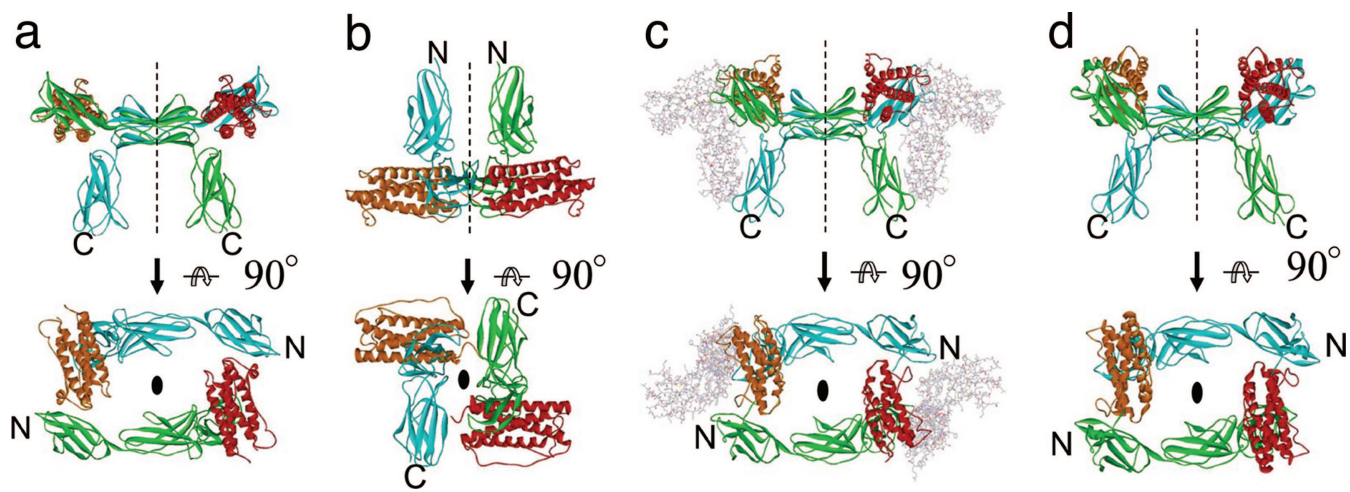

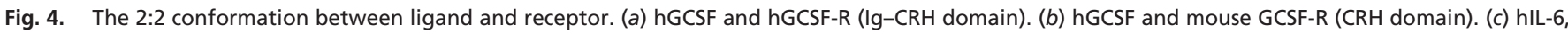

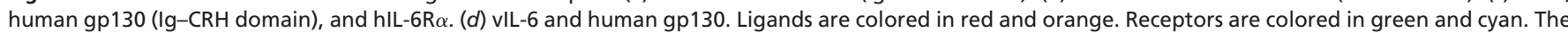
IL-6R $\alpha$ molecules are drawn by wire model. Dashed lines and ellipse show twofold axis.

GCSFs and CRH domains (omitting the Ig-like domain), the association scheme of the hGCSF/mCRH complex is completely different from that of the hGCSF/hIg-CRH complex determined here. The BC domains in the hGCSF/mCRH structure are situated antiparallel with a $\mathrm{C}$ terminus distance of $79 \AA$, whereas the $\mathrm{BC}$ domains in the $\mathrm{hGCSF} / \mathrm{hIg}-\mathrm{CRH}$ structure are parallel with a $\mathrm{C}$ terminus distance of $\approx 70 \AA$.

Because the molecular mass of the sample used for crystallization was confirmed to be $111,600 \mathrm{Da}$ by light scattering analysis (21), characteristic of a 2:2 complex composed of two hGCSFs and two Ig-CRHs, the 2:2 complex formed by means of cross-over interactions between the Ig-like domain of hGCSF-R and the neighboring hGCSF, is a signaling complex seen in the solution state. Additional data support a 2:2 signaling complex stoichiometry for hGCSF/hIg-CRH. Isothermal titration calorimetry studies (10) of the binding of hGCSF to Ig-CRH domains of hGCSF-R suggested that the total buried surface area was 5,700-6,000 $\AA^{2}$. The total buried surface area for the 2:2 signaling complex in the x-ray structure reported here is calculated to be $5,038 \AA^{2}$, based on a contribution of $1,375 \AA^{2}$ from site II and 1,144 $\AA^{2}$ from site III.

From the observations discussed above, as well as the fact that the Ig-like domain strongly contributes to proliferation response (22), the association of two hGCSF/mCRHs without the Ig-like domain is unlikely to represent a functional signaling complex, but rather, a crystallographic association of two units of hGCSF/mCRH in the crystal lattice. The 2:2 complex of hGCSF/hIg-CRH determined here likely represents the functional signaling complex of GCSF-R.

The extracellular region of GCSF-R showed similarity to that of gp130 (26\% amino acid identity). Gp130 is known as a shared signal transducing receptor for several cytokines (e.g., IL-6, leukemia inhibitory factor, and oncostatin) and also has an Ig-like, a CRH, and three fibronectin type III domains in the extracellular region (23). There are some reports detailing chimeric receptor analysis between GCSF-R and gp130. GCSF was reported to bind to the chimeric GCSF-R having an Ig-like domain from gp130 but could not transduce a signal (18). GCSF could not bind to a chimeric receptor having both Ig-like and CRH domains from gp130 (24). These phenomena also were observed in the case of IL-6 and human chimeric gp130s. IL-6 bound to chimeric gp130 having the Ig-like domain from GCSF-R but lost activation $(24,25)$. These phenomena support an activation mechanism based on the 2:2 structure of hGCSF/hIg-CRH in which both Ig-like and CRH domains must simultaneously interact with GCSF.

Garcia and coworkers (13) have already determined the hexameric (2:2:2) signaling complex structure consisting of two sets of 1:1:1 complex from IL-6, CRH domain of hIL-6R $\alpha$, and Ig-CRH domain of human gp130 (Fig. 4c) and the tetrameric (2:2) complex structure consisting of two sets of 1:1 complex from vIL-6 and
Ig-CRH domain of human gp130 (Fig. 4d) (14). Both sets of complexes are related by means of twofold crystallographic symmetry and form a symmetrical arrangement tethered with each Ig-like domain of gp130 (Figs. $4 c$ and $d$ ). The tertiary structure of hGCSF/hIg-CRH also exhibits two sets of 1:1 complex from hGCSF and Ig-CRH domain of hGCSF-R, similar to the two sets of 1:1 association between IL-6 and gp130.

The primary and secondary structures of the ligands (GCSF, hIL-6, and vIL-6), receptors (Ig-CRHs from GCSF-R and gp130), and residues involved in ligand-receptor interaction (as determined by the x-ray structure determination) are shown in Fig. 2. The rms deviations between the Ig-CRH domains (Ig-like, Cys-3-Ala-96; BN, Gly-97-Asp-200; BC, Val-201-Thr-306) of hGCSF-R and gp130 in hIL-6 or vIL- 6 complex, calculated by using $\mathrm{C}^{\alpha}$ atoms, are

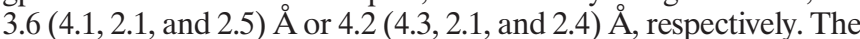
overall arrangements of the ligands and the Ig-like and CRH domains are shared between the signaling complexes of hGCSF-R and human gp130.

The residues of GCSF and IL-6 involved in the site II interactions are partly conserved in their primary and tertiary structure (as shown in red in Fig. 2), but the locations of the ligands are slightly different. The relative location of GCSF seen in the structure of $\mathrm{hGCSF} / \mathrm{hIg}-\mathrm{CRH}$ (and $\mathrm{hGCSF} / \mathrm{mCRH}$ ) is rotated $\approx 20^{\circ}$ based on the position of $\alpha \mathrm{A}$ (Fig. $5 a$ ) in comparison with the locations of hIL-6 and vIL-6. The positional shift of GCSF resulted in additional interactions with Arg-288 and Leu-291 in the loop of BC domain of GCSF-R (Fig. 5a). Arg-288 is an essential residue for the biological and binding activities of GCSF-R (16-18). For the IL- 6 recognition, Trp-142 and His-145 (Thr-144 in vIL-6 complex) in the BN domain of gp130 are involved; those are replaced with the seven-residue loop and were not involved in GCSF recognition. These changes in ligand binding contribute to improved specificity of ligand-receptor interaction at site II.

Both hIL- 6 and vIL- 6 exhibits site III interactions by means of binding to Ig-like domains (Fig. 2). The details of site-III interaction at the N-terminal region of the receptor differ substantially. Eight residues in gp130 interact with hIL-6 (six residues with vIL-6) and are distributed over the region Leu-2-His-49 in gp130, whereas only Cys-3 (disulfide bonded to Cys-29) is involved in the ligand binding in hGCSF-R (Fig. 5b). This difference is more obvious in the contribution of the $\mathrm{N}$ terminus region of gp130. The $\mathrm{N}$-terminal region (Leu-2-Pro-5) extends between the A-B loop and the $\mathrm{N}$-terminal of $\alpha \mathrm{D}$ in IL-6 to form hydrogen-bonding interactions with Glu-58-Asn-59 in hIL-6 (Ala-44-Ile-46 in vIL-6) (Fig. 5b). This region is replaced by a short helix (Val-49-Leu-55) in GCSF and contributes to receptor binding (Fig. 5b). This difference appears to be responsible for the unique specificity of ligandreceptor binding at site III. 


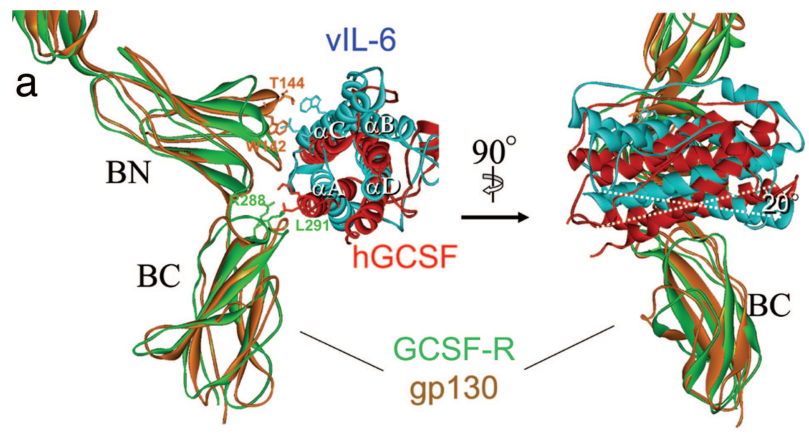

b
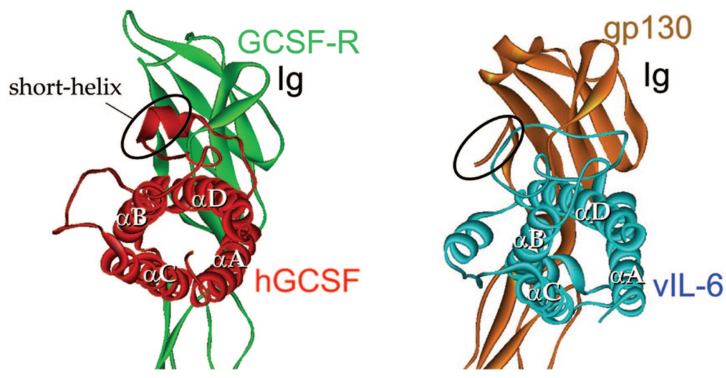

Fig. 5. Structural comparison of GCSF-R complex and gp130 complex. (a) Site II. (b) Site III. Complexes are superimposed in a and separated in $b$. The GCSF, GCSF-R, IL-6, and gp130 molecules are colored in red, green, cyan, and orange, respectively. Related residues of specificity are drawn by stick model.

Thus, the ligand activation scheme of the hGCSF-R has been reevaluated in light of the tertiary structure analysis of the signaling complex of hGCSF/hIg-CRH. Although there are important differences in site II and III interaction, the overall activation scheme of GCSF-R induced by GCSF is similar to that of gp130 induced by IL-6. Because of this structural similarity, the possibilities of site I interaction (to $\alpha$ chain receptor) in GCSF should be mentioned.
The residues involved in the site I interaction in hIL-6 2:2:2 complex is shown as green in Fig. 2. The site I interaction to IL-6R $\alpha$ is mainly formed by $\alpha \mathrm{D}$ of IL-6. Because two Args (Arg-166 and -169 in hGCSF) in $\alpha \mathrm{D}$ are conserved between hGCSF and hIL-6, and the model of the 2:2:2 (hGCSF/hGCSF-R/hIL-6R $\alpha$ ) complex can be built without any steric hindrance, the hIL-6R $\alpha$ binding to GCSF/ hIg-CRH was tested by using surface plasmon resonance. It was found that hIL-6R $\alpha$ exhibited moderate affinity $\left(K_{\mathrm{D}}=1.02 \times 10^{-7}\right.$ $\mathrm{M})$ for hGCSF/hIg-CRH complex that is comparable with the affinity between hIL-6 and hIL-6R $\alpha$ (13). Although the Ala mutations of Arg-166 and -169 in hGCSF did not show any effect on proliferation activity (17), the structural information for hGCSF/hGCSF-R might suggest other functions of IL- $6 \alpha$ in the signaling pathway of GCSF-R.

\section{Materials and Methods}

Protein Expression, Purification, and Crystallization. Protein preparation and crystallization of hGCSF and Ig-CRH domains of hGCSF-R were performed as described in ref. 21 . Briefly, crystals were grown at $293 \mathrm{~K}$ in hanging drops using $1.5 \mathrm{mg} / \mathrm{ml}$ protein solution over $0.2 \mathrm{M}$ ammonium phosphate, and were identified as space group $\mathrm{P} 3{ }_{2} 21$ with unit-cell parameters $a=b=134.8, c=$ 105.7 $\AA$. The Matthews coefficient was $4.9 \AA^{3} /$ Da assuming one 1:1 complex in the asymmetric unit, corresponding to unusually high solvent content $(75 \%)$.

Data Collection and Structure Determination. Data collection measurements were performed at BL38B1 and BL41XU in SPring-8. Reflections were collected on Area Detector Systems Corporation (Poway, CA) Quantum-4R (BL38B1) and MAR-Research (Hamburg) Mar165 (BL41XU) charge-coupled device detectors at $100 \mathrm{~K}$ from crystals that were soaked in a solution containing $0.2 \mathrm{M}$ ammonium phosphate and $30 \%$ glycerol as a cryoprotectant and then flash-frozen in a nitrogen-gas stream. Intensity data were processed by using HKL2000 (26). The native data set was integrated and scaled to $2.8-\AA$ resolution. Data collection, phasing, and refinement statistics are summarized in Tables 2 and 3, respectively.

Table 2. Data collection and phasing added to molecular replacement

\begin{tabular}{|c|c|c|c|}
\hline & Native & $\mathrm{Se}^{*}$ & $\mathrm{Hg}$ * \\
\hline \multicolumn{4}{|l|}{ Data collection } \\
\hline Beamline & SPring8 [BL38B1] & SPring8 [BL41XU] & SPring8 [BL41XU] \\
\hline Wavelength, Å & 1.0000 & 0.9791 & 1.0083 \\
\hline Resolution (highest shell), Å & $2.80(2.90-2.80)$ & $3.40(3.52-3.40)$ & $3.20(3.31-3.20)$ \\
\hline Total reflections & 153,280 & 157,583 & 147,841 \\
\hline Unique reflections & 25,214 & 28,890 & 33,684 \\
\hline Mosaicity & 0.65 & 0.78 & 0.28 \\
\hline Redundancy & $5.9(2.2)$ & $5.5(3.9)$ & $4.5(2.7)$ \\
\hline$I / \sigma(I)$ & $20.3(1.9)$ & $12.8(1.2)$ & $9.4(1.7)$ \\
\hline Completeness, \% & $91.0(78.0)$ & $98.2(85.0)$ & $95.7(74.6)$ \\
\hline$R_{\text {merge }}^{\dagger}$ & $0.058(0.355)$ & $0.083(0.430)$ & $0.088(0.211)$ \\
\hline \multicolumn{4}{|l|}{ MIR phasing (3.50 A) } \\
\hline$R_{\text {iso }}{ }^{\ddagger}$ & - & 0.104 & 0.275 \\
\hline$R_{\text {anom }} \S$ & - & 0.072 & 0.072 \\
\hline No. of sites & - & 3 & 1 \\
\hline \multicolumn{4}{|l|}{ Phasing power ${ }^{\Uparrow}$} \\
\hline Centric, acentric & - & $0.18,0.27$ & $0.68,0.95$ \\
\hline$R_{\text {cullis }}$ centric, acentric & - & $0.97,0.99$ & $0.83,0.88$ \\
\hline Figure of merit & & 0.33 & \\
\hline \multicolumn{4}{|c|}{$\begin{array}{l}{ }^{\dagger} R_{\text {merge }}=\Sigma \mid I(h)-\langle I(h)\rangle / \Sigma I(h) \text {, where }\langle I(h)\rangle \text { is the mean value of the reflection } h \text { for all measurements of } I(h) \text {. } \\
{ }^{\ddagger} R_{\text {iso }}=\Sigma\left|F_{\mathrm{PH}}-F_{\mathrm{P}}\right| / \Sigma F_{\mathrm{PH}}, \text { where } F_{\mathrm{PH}} \text { and } F_{\mathrm{P}} \text { are the derivatives and native structure factor amplitude, respectively. } \\
{ }^{\S} R_{\text {anom }}=\Sigma\left|I^{+}(h)-I^{-}(h)\right| / \Sigma\left(I^{+}(h)+I^{-}(h)\right) \text {, where } I^{+}(h) \text { and } I^{-}(h) \text { are the Bijvoet pairs of } I(h) \text {. } \\
\text { "l Phasing power }=\left\langle F_{\mathrm{H}}\right\rangle / E \text {, where }\left\langle F_{\mathrm{H}}\right\rangle \text { is the mean of calculated heavy atom structure factor amplitude and } E \text { is the } \\
\text { rms lack of closure error. }\end{array}$} \\
\hline
\end{tabular}


Table 3. Summary of refinement statistics

\begin{tabular}{lc} 
Refinement & Value \\
\hline Resolution (highest shell), $\AA$ & $2.80(2.87-2.80)$ \\
Used reflections & 23,837 \\
$R_{\text {cryst }}{ }^{*}$ & $0.253(0.439)$ \\
$R_{\text {free }}{ }^{\dagger}$ & $0.284(0.439)$ \\
No. of atoms & 3,647 \\
Protein & 3,605 \\
Sugar chain & 28 \\
Water & 14 \\
Mean B value, overall, ${ }^{2}$ & 68.0 \\
GCSF & 71.9 \\
Ig-like domain of GCSF-R & 92.8 \\
BN domain of GCSF-R & 54.8 \\
BC domain of GCSF-R & 66.3 \\
rms deviation & \\
Bond length, $\AA^{2}$ & 0.037 \\
Bond angles, & \\
Ramachandran angles, $\%$ & 2.98 \\
Most favored & 78.0 \\
Allowed & 19.9
\end{tabular}

${ }^{*} R_{\text {cryst }}=\Sigma|| F_{\text {obs }}|-| F_{\text {calc }}|| \Sigma\left|F_{\text {obs }}\right|$, where $F_{\text {obs }}$ and $F_{\text {calc }}$ are observed and calculated structure factor amplitude, respectively.

${ }^{\dagger} R_{\text {free }}$ is the same as $R_{\text {cryst, }}$ but for a $5 \%$ subset of all reflections.

Initial phase information for hGCSF and $\mathrm{CRH}$ domain of hGCSF-R was obtained by molecular replacement (MR) with PHASER (27) using the coordinates of the 1:1 complex of hGCSF and CRH domain of mouse GCSF-R (Protein Data Bank ID code 1CD9). Because electron densities calculated using the initial phase information were insufficient for chain tracing of the Ig-like domain of hGCSF-R, derivatives were obtained by soaking crystals in $1 \mathrm{mM}$ thimerosal or by expression of hGCSF in medium containing

1. Nagata, S., Tsuchiya, M., Asano, S., Kaziro, Y., Yamazaki, T., Yamamoto, O., Hirata, Y., Kubota, N., Oheda, M., Nomura, H., et al. (1986) Nature 319, 415-418.

2. Metcalf, D. (1990) Nature 339, 27-30.

3. Welte, K., Gabrilove, J., Bronchud, M. H., Platzer, E. \& Morstyn, G. (1996) Blood 88, 1907-1929.

4. Fukunaga, R., Ishizaka-Ikeda, E., Seto, Y. \& Nagata, S. (1990) Cell 61, 341-350.

5. Fukunaga, R., Seto, Y., Mizushima, S. \& Nagata, S. (1990) Proc. Natl. Acad. Sci. USA 87, 8702-8706.

6. Avalos, B. R. (1996) Blood 88, 761-777.

7. Hiraoka, O., Anaguchi, H. \& Ohta, Y. (1995) Biosci. Biothechnol. Biochem. 59, 2351-2354.

8. Horan, T. P., Wen, J., Narhi, L., Parker, V., Garcia, A., Arakawa, T. \& Philo, J. (1996) Biochemistry 35, 4886-4896.

9. Hiraoka, O., Anaguchi, H. \& Ohta, Y. (1994) FEBS Lett. 356, 255-256.

10. Mine, S., Koshiba, T., Honjo, E., Okamoto, T., Tamada, T., Maeda, Y., Matsukura, Y., Horie, A., Ishibashi, M., Sato, M., et al. (2004) Biochemistry 43, 2458-2464.

11. Aritomi, M., Kunishima, N., Okamoto, T., Kuroki, R., Ota, Y. \& Morikawa, K. (1999) Nature 401, 713-717.

12. Ishibashi, M., Tokunaga, H., Arakawa, T. \& Tokunaga, M. (2001) Protein Express. Purif. 21, 317-322.

13. Boulanger, M. J., Chow, D. C., Brevnova, E. E. \& Garcia, K. C. (2003) Science 300, 2101-2104

14. Chow, D., He, X., Snow, A. L., Rose-John, S. \& Garcia, K. C. (2001) Science 291, 2150-2155.

15. Haniu, M., Horan, T., Arakawa, T., Le, J., Katta, V., Hara, S. \& Rohde, M. F. (1996) Biochemistry 35, 13040-13046.

16. Reidhaar-Olson, J. F., De Souza-Hart, J. A. \& Selick, H. E. (1996) Biochemistry 35, 9034-9041.

17. Young, D. C., Zhan, H., Cheng, Q. L., Hou, J. \& Matthews, D. J. (1997) Protein Sci. 6, 1228-1236.

18. Layton, J. E., Shimamoto, G., Osslund, T., Hammacher, A., Smith, D. K., Treutlein, H. R. \& Boone, T. (1999) J. Biol. Chem. 274, 17445-17451. selenomethioine using a nonauxotroph strain for methionine (wild strain) (28). Data of derivatives were collected under absorption peak wavelength based on fluorescence profiles. Electron densities calculated from multiple isomorphous replacement with anomalous scattering (MIRAS) phasing with MLPHARE (29) using selenium and mercury derivatives had enough quality to permit partial tracing of the Ig-like domain. Further density modifications were performed by solvent flattening with DM (30) and a combination of MIRAS and MR phases with SIGMAA (31). An atomic model was built with TURBO-FRODO (32). Crystallographic refinement was carried out by energy minimization and simulated annealing with molecular dynamics by using CNS (33) and by maximum likelihood refinement using REFMAC (34), with manual rebuilding at each refinement cycle.

The final model, including 468 residues (hGCSF, Ser-7-Pro-174; hGCSF-R, Cys-8-Glu-314, except for Ser-220-Pro-226), one Nlinked sugar chain (including two $N$-acetyl-D-glucosamine) at Asn111 in hGCSF-R and 14 waters, refined to a crystallographic $R$ factor of $25.3 \%$ (free $R$-factor $=28.4 \%$ ), using all 23,837 reflections to $2.8-\AA$ resolution. The stereochemistry of the refined atomic model analyzed by PROCHECK (35) showed that $98 \%$ of the mainchain atoms fall within the allowed regions of the Ramachandran plot and the side-chain geometry for the $\chi-1$ and $\chi$ - 2 stereochemical parameters is inside expected regions at this resolution.

We thank S. Mine, T. Koshiba, Y. Matsukura, A. Horie, M. Sato, M. Azuma, and M. Adachi for assistance; H. Sakai, K. Hasegawa, and M. Kawamoto for data collection with synchrotron radiation at SPring-8 (Proposals 2003A-0221, 0222, and 0749); N. Shibata for suggestions about phasing; and $\mathrm{M}$. Blaber for suggestions about manuscript preparation. This work was supported in part by the Pilot Applied Research Project for the industrial use of space (to R.K.) from Japan Space Utilization Promotion Center and Japan Aerospace Exploration Agency, the Protein 3000 Project (to R.K.), and Grant-in-Aid for Scientific Research (C) 17570101 (to T.T.) from the Ministry of Education, Culture, Sports, Science, and Technology (Japan).

19. Layton, J. E., Iaria, J., Smith, D. K. \& Treutlein, H. R. (1997) J. Biol. Chem 272, 29735-29741.

20. Layton, J. E., Hall, N. E., Connell, F., Venhorst, J. \& Treutlein, H. R. (2001) J. Biol. Chem. 276, 36779-36787.

21. Honjo, E., Tamada, T., Maeda, Y., Koshiba, T., Mine, S., Matsukura, Y., Okamoto, T., Ishibashi, M., Tokunaga, M. \& Kuroki, R. (2005) Acta Crystallogr. F 61, 788-790.

22. Fukunaga, R., Ishizaka-Ikeda, E., Pan, C. X., Seto, Y. \& Nagata, S. (1991) EMBO J. 10, 2855-2865.

23. Bravo, J. \& Heath, J. K. (2000) EMBO J. 19, 2399-2411.

24. Hammacher, A., Wijdenes, J., Hilton, D. J., Nicola, N. A., Simpson, R. J. \& Layton, J. E. (2000) Biochem. J. 345, 25-32.

25. Ward, L. D., Howlett, G. J., Discolo, G., Yasukawa, K., Hammacher, A., Moritz, R. L. \& Simpson, R. J. (1994) J. Biol. Chem. 269, 23286-23289.

26. Otwinoski, Z. \& Minor, W. (1997) Methods Enzymol. 276, 307-326.

27. Storoni, L. C., McCoy, A. J. \& Read, R. J. (2004) Acta Crystallogr. D 60, 432-438.

28. Tamada, T., Feese, M. D., Ferri, S. R., Kato, Y., Yajima, R., Toguri, T. \& Kuroki, R. (2004) Acta Crystallogr. D 60, 13-21.

29. Otwinoski, Z. (1991) in Daresbury Study Weekend Proceedings, eds. Wolf, W., Evans, P. R. \& Lesile, A. G. M. (SERC Daresbury Laboratory, Warrington, U.K.), pp. $80-86$.

30. Cowtan, K. (1994) Joint CCP4 and ESF-EACBM Newslett. Protein Crystallogr. 31, 34-38.

31. Read, R. J. (1986) Acta Crystallogr. A 42, 140-149.

32. Jones, T. A. (1978) J. Appl. Crystallogr. 11, 268-272.

33. Brünger, A. T., Adams, P. D., Clore, G. M., DeLano, W. L., Gros, P., Grosse-Kunstleve, R. W., Jiang, J.-S., Kuszewski, J., Nilges, N., Pannu, N. S., et al. (1998). Acta Crystallogr. D 54, 905-921.

34. Murshudov, G. N., Vagin, A. A. \& Dodson, E. J. (1997) Acta Crystallogr. D 53, 240-255.

35. Laskowski, R. A., MacArtur, M. W., Moss, D. S. \& Thornton, J. M. (1993) J. Appl. Crystallogr. 26, 283-291. 